\title{
Enhanced Degradation of Organic Pollutants with Microwave-induced Plasma-in-liquid (MPL): Case of Flame Retardant Tetrabromobisphenol-A in Alkaline Aqueous Media
}

\author{
Satoshi Horikoshi ${ }^{1 *}$, Seiya Sawada ${ }^{1}$, Akihiro Tsuchida ${ }^{1}$, and Nick Serpone ${ }^{2}$ \\ ${ }^{1}$ Department of Materials and Life Sciences, Faculty of Science and Technology, Sophia University, 7-1 Kioicho, Chiyodaku, Tokyo 102-8554, \\ JAPAN \\ ${ }^{2}$ PhotoGreen Laboratory, Dipartimento di Chimica, Università di Pavia, Via Taramelli 12, Pavia 27100, ITALIA
}

\begin{abstract}
We report the enhanced degradation of a widely used brominated flame retardant, tetrabromobisphenol-A (TBBPA), which is soluble only in organic solvents and strongly alkaline solutions, where most advanced oxidation processes (AOPs) for such substrates tend to be rather inefficient. We further report an environmentally friendly method (microwave-induced plasma-in-liquid; MPL) that operates efficiently in alkaline aqueous media without the need for organic solvents to enhance the solubility of TBBPA in water. The enhanced debromination and almost complete mineralization of TBBPA under alkaline conditions occurs within 20 min of MPL irradiation. This method, which is a new member of the AOP family, provides a simple and green approach to detoxify aqueous media contaminated with TBBPA, which requires only electric power and neither catalysts nor oxidizing agents. Several intermediate species have been identified by liquid chromatography/mass spectrometry (LC-MS), following events that involved reactive oxygen species (ROSs) such as $\cdot \mathrm{OH}$, whose first task was to approach the substrate at carbon atoms bearing the highest electron densities.
\end{abstract}

Key words: microwave-induced plasma-in-liquid, tetrabromobisphenol-A, AOP, degradation, semiconductor microwave generator

\section{Introduction}

One of the first applications of advanced oxidation processes (AOPs) was reported in 1987 by Glaze ${ }^{1)}$, who proposed that such processes involve the generation of $\cdot \mathrm{OH}$ in sufficient quantity to affect water purification. Several AOPs have been described since, many of which involve use of the powerful oxidizing hydroxyl radical $\cdot \mathrm{OH}$ that can oxidize and degrade organic pollutants from contaminated air and polluted aqueous ecosystems. These AOPs are termed advanced, because the chemical reactions are essentially the same except that they occur considerably faster(billions of times) vis-à-vis reactions that occur if the pollutants were exposed solely to a natural environment ${ }^{2)}$. Other AOPs include UV/O $\mathrm{O}_{3}, \mathrm{UV} / \mathrm{H}_{2} \mathrm{O}_{2}$, Fenton, photo-Fenton, non-thermal plasmas, sonolysis, photocatalysis, radiolysis, and supercritical water oxidation processes ${ }^{3-7)}$. However, newer approaches to achieve advanced oxidation processes have been lacking in recent years.

The flame retardant tetrabromobisphenol-A (TBBPA) is found worldwide in polymeric substrates, rubber products, and fiber products ${ }^{8}$. Because of its high lipophilicity and persistency, TBBPA is ubiquitous in most environmental matrices including wastewaters, soils, sewage sludge, source waters, and aquatic sediments ${ }^{9)}$. Additionally, TBBPA has also been detected in fish, human plasma serum, and in human breast milk. Most notably, TBBPA is a potential endocrine disruptor that can induce disruption of thyroid homeostasis and estrogen signaling ${ }^{9)}$. Sun and coworkers reported on the fate and metabolites of 14CTBBPA in a submerged soil with an anoxic/oxic interface with and without planted rice (Oryza sativa) and reed (Phragmites australis) seedlings ${ }^{10)}$. The accelerated transformation of TBBPA and its reduction in bound-residue formation in planted versus unplanted submerged soil

*Correspondence to: Satoshi Horikoshi, Department of Materials and Life Sciences, Faculty of Science and Technology, Sophia University, 7-1 Kioicho, Chiyodaku, Tokyo 102-8554, JAPAN

E-mail: horikosi@sophia.ac.jp

Accepted December 30, 2019 (received for review December 23, 2019)

Journal of Oleo Science ISSN 1345-8957 print / ISSN 1347-3352 online

http://www.jstage.jst.go.jp/browse/jos/ http://mc.manusriptcentral.com/jjocs 
demonstrates the potential of wetland plants, especially reed, in the remediation of TBBPA-contaminated soils, although sometime it is necessary for the process to occur. Hence, it is highly desirable to develop effective treatment methods to remove TBBPA under aqueous alkaline conditions prior to its discharge into the environment.

The report published by Clements in $1987^{11)}$ on plasma generation by a high-voltage pulse discharge in water (material changed to a plasma state) marked the leading edge of research on plasma-in-liquid (PL). After its publication, several researchers in the fields of electricity and physics focused their attention to the generation of plasma in liquids. Accordingly, various studies into the physical elucidation of PL have been carried out since in numerous research sectors. Generation of an underwater streamer discharge with a DC and AC electrical power supply - even in aqueous media - necessitates an electrical power of 50 to $400 \mathrm{kV}$ and a current of $100 \mathrm{~A}$ or more ${ }^{12)}$. Industrially, however, it is difficult to achieve and maintain a steadystate electrical power of this magnitude from the viewpoint of stability and energy savings. Nonetheless, Nomura and colleagues succeeded in generating PL even under energysaving conditions by using radiofrequency or microwave power $^{13)}$. Unlike other techniques, it was not necessary to use an electrolyte solution. Radiofrequency- or microwaveinduced plasma-in-liquid (MPL) can be maintained in water across a wide range of conductivities (from 0.2 to $7000 \mathrm{mS}$ $\mathrm{m}^{-1}$ ). In a previous study, we succeeded in generating plasma over a long period of time in a stable manner by irradiating with microwaves in a pulsed state and in a depressurized aqueous medium ${ }^{14)}$. Recent plasma applications into various other fields have witnessed significant progress in fields of nanomaterial synthesis ${ }^{15}$, hydrogen generation $^{16)}$, and in the treatment of Microcystis aeruginosa $a^{17)}$.

The plasma-in-liquid (PL) method is a technology in which plasma is generated inside bubbles in a liquid ${ }^{12)}$. Various oxidizing and radical species are generated in such plasma from the UV light derived either from the plasma or from the plasma energy ${ }^{12)}$. An earlier study ${ }^{18)}$ demonstrated that the MPL method can rapidly process the degradation of perfluorooctanoic acid (PFOA), a rather difficult substrate to degrade with major advanced oxidation processes (AOP). Undoubtedly, the MPL method could also decompose TBBPA.

This study describes the decomposition of TBBPA under alkaline conditions by the PL method, whose efficiency was assessed from the decrease of the concentration of TBBPA through debromination events and loss of total organic carbon (TOC), thereby asserting the technique as an effective AOP method. Accordingly, we propose the submerged MPL method be added as another AOP among the methods currently being used.

\section{Experimental Procedures}

\subsection{Microwave-induced plasma-in-liquid (MPL) device}

A photograph and a schematic illustration of the MPL device are displayed in Fig. 1a and 1b, respectively. Figure 1c displays a photograph of the MPL. The microwave device was constructed using an Ampreon M2A-R semiconductor generator that emits either continuous or pulsed microwaves (maximal power, $1200 \mathrm{~W}$; microwave frequency, $2.45 \mathrm{GHz}$ ), together with an isolator (air cooling device), a power monitor, a three-stub tuner, and a shortcircuit plunger. The liquid medium was continuously irradiated with microwaves through the tungsten electrode antenna (diameter, $1.0 \mathrm{~cm}$; length, $20.0 \mathrm{~cm}$ ). Metal cones were used in the waveguide to focus microwaves onto the tip of the antenna electrode, through which the microwaves irradiate the aqueous solution and thus generate the MPL (Fig. 1b). The tungsten antenna was isolated from both the reactor and the waveguide using a ceramic spacer, such that the microwave irradiates only the liquid. The cylindrically shaped reactor consists of a quartz vessel (diameter, $3.20 \mathrm{~cm}$; length, $16.0 \mathrm{~cm}$ ) and was fabricated such that the pressure could be reduced with a water aspirator through the top of the reactor. The reaction vessel was fixed to a tungsten pedestal with a metal spacer within the waveguide. The reason for the decompression is that nonthermal plasma can be generated, and deterioration of the electrode antenna is prevented under conditions of reduced pressure and application of pulsed microwaves. The irradiating microwaves were adjusted by the threestub tuner in such a way to avoid reflected waves. The microwave irradiation consisted of cycles of 1.33-ms irradiation pulses and 0.34-ms dark pauses. The optimal microwave irradiation conditions are described in our previous study ${ }^{14)}$. The generated plasma glows white and yellow; the clear water appears to emit white and blue light (Fig. 1c).

\subsection{Degradation of tetrabromobisphenol-A}

High-purity grade tetrabromobisphenol-A (TBBPA) was obtained from Wako Pure Chemical Industries, Ltd. The aqueous TBBPA solution ( $50 \mathrm{~mL} ; 0.10 \mathrm{mM} \approx 54.4 \mathrm{mg} \mathrm{L}^{-1}$ ) was placed in the quartz reactor and subsequently subjected to irradiation with the generated MPL. The solution was adjusted to $\mathrm{pH}=9.0,11.0$, and 13.0 with sodium hydroxide. TBBPA dissolves only in alkaline media; and at lower $\mathrm{pH}$, TBBPA tends to precipitate. Samples of the decomposed TBBPA solution were collected at various time intervals and subsequently analyzed to assess the extent of debromination of the TBBPA $\left(\mathrm{Br}^{-}\right.$ion concentration of irradiated samples was measured to calculate the extent of debromination) using a Thermo Scientific ${ }^{\mathrm{TM}}$ Dionex $^{\mathrm{TM}}$ Integrion $^{\mathrm{TM}}$ HPIC $^{\mathrm{TM}}$. The loss of total organic carbon(TOC) was determined using a Shimadzu TOC-L analyzer. The emission spectrum of the MPL with sample solution was measured 


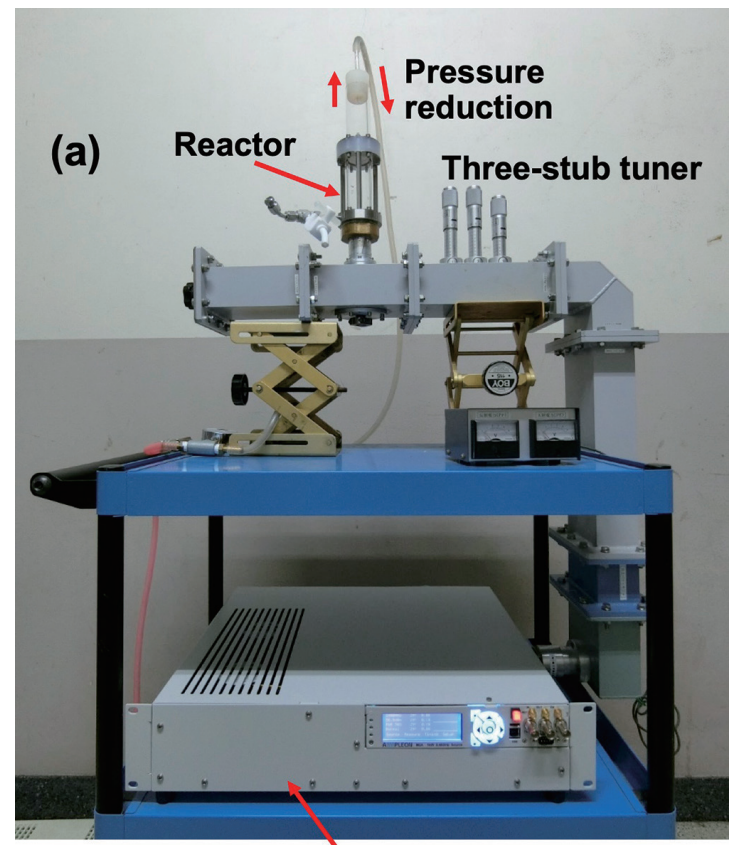

Semiconductor generator
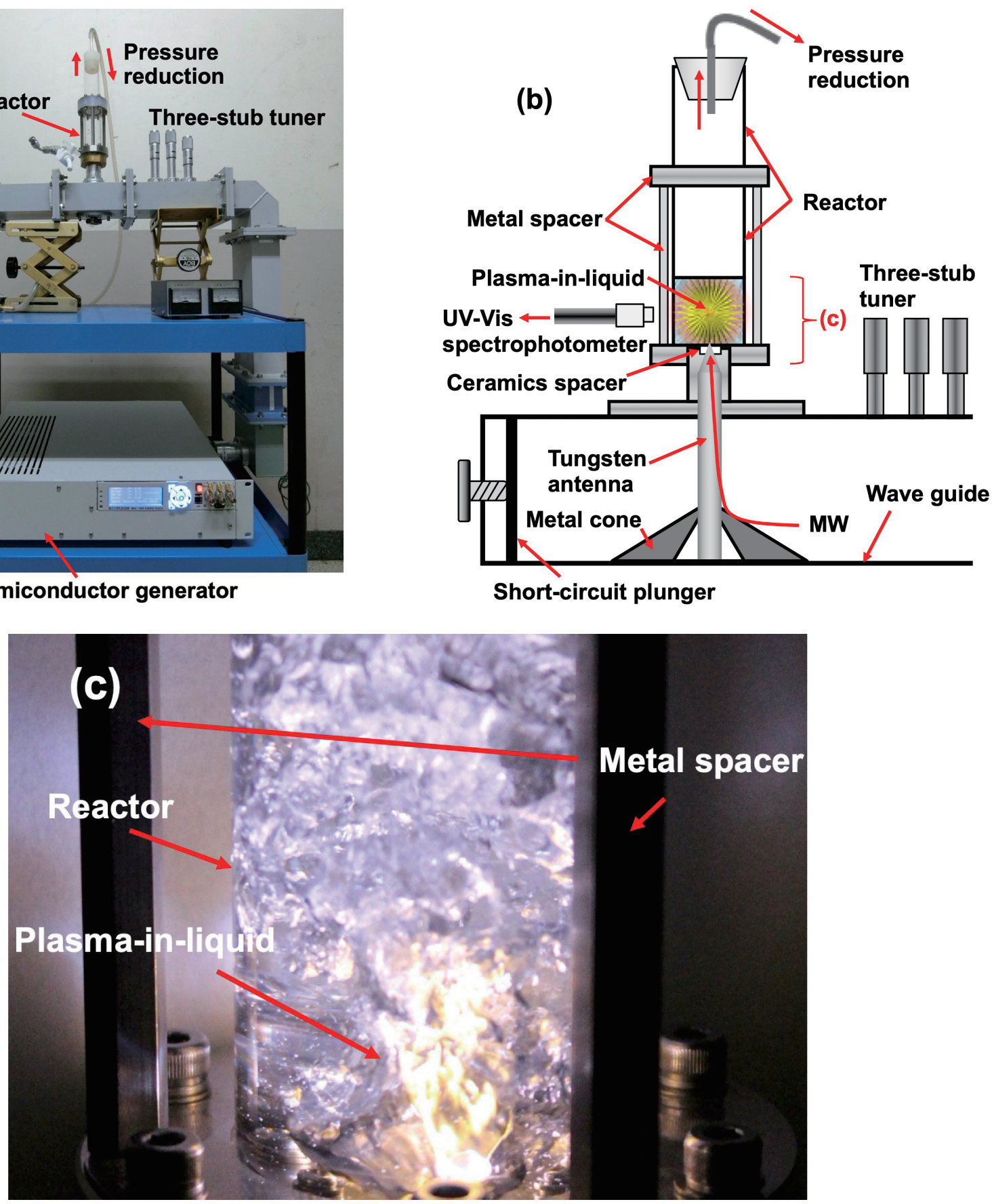

Fig. 1 (a) Photograph and (b) schematic illustration of the microwave-induced plasma-in-liquid (MPL) device; (c) photograph of the generated plasma-in-liquid in the reactor.

through an optical fiber using a UV-Vis spectrophotometer (MayaPro2000, Ocean Insight). The measured spectrum was analyzed using the PLASUS SpecLine software(Ver. 2.1) from the Ocean Optics Company.

Computer simulations were carried out using a CAChe Worksystem version 3.2 (Fujitsu Co. Ltd) implemented on an Intel P-III and Windows 7 system. Frontier electron densities, used to ascertain positions of radical attack on the heteroaromatic substrate, were also calculated using the MOPAC/PM3 wavefunction. A geometrical configuration was determined by pre-optimization calculation in the Mechanics using augmented MM3, followed by geometrically optimized calculation in MOPAC using PM3 parameters; solvation effects in water were also simulated using $\mathrm{COSMO}^{19)}$.

Intermediate species formed during the debromination 
and mineralization of TBBPA were identified by direct injection into the mass spectral detector of a JEOL Ltd., JMS-T100LC LC-TOFMS (electrospray ionization: ESI) system, operated in the negative ion mode, where the orifice voltage was $30 \mathrm{~V}$. The eluent was a solution of methanol. The LC column was not used in this study. The spectrometer was scanned from $\mathrm{m} / z=100$ to 1000 for recording mass spectra.

\section{Results and Discussion}

\subsection{Degradation of tetrabromobisphenol-A}

The time course of the debromination of aqueous TBBPA solutions under various alkaline conditions $(\mathrm{pH}=9.0,11.0$, and 13.0) was examined with the MPL system(Fig. 1a). Complete debromination occurred $(\sim 100 \%)$ when the process was carried out at $\mathrm{pH} 13.0$ for an irradiation time of $20 \mathrm{~min}$, whereas at $\mathrm{pH}$ values of 11.0 and 9.0 the extent of debromination was $87 \%$ and $83 \%$, respectively (see Fig. 2a). Debromination followed first-order kinetics: $k=0.105$ $\pm 0.011 \mathrm{~min}^{-1}(\mathrm{pH} 13.0), k=0.078 \pm 0.009 \mathrm{~min}^{-1}(\mathrm{pH} 11.0)$, and $k=0.078 \pm 0.010 \mathrm{~min}^{-1}(\mathrm{pH} 9.0)$. By comparison, the extent of mineralization of TBBPA was nearly complete $(99.9 \%$ ) within 20 min of irradiation at $\mathrm{pH} 13.0$, while the extent of TOC loss was $81 \%$ at $\mathrm{pH} 11.0$ and $76 \%$ at $\mathrm{pH} 9.0$, as shown in Fig. 2b. Hence, the optimal condition to degrade and mineralize the TBBPA flame retardant is in aqueous alkaline media at $\mathrm{pH}$ 13.0.

The degradation of TBBPA has been frequently examined by other AOP. Accordingly, it is relevant to compare the degradation efficiency of TBBPA by the presently employed MPL treatment method with those employed earlier (see right). The decomposition of TBBPA in aqueous media has also been carried out by $\mathrm{O}_{3}$ oxidation ${ }^{6)}$.

The concentration of TBBPA used in earlier studies was about $50 \mathrm{ppm}\left(50 \mathrm{mg} \mathrm{L}^{-1}\right)$, which is slightly lower than the concentration used in the present study. For a reaction time of $25 \mathrm{~min}$, the TBBPA removal was $66.7 \%$ by $\mathrm{O}_{3}$, after which removal of TBBPA by $\mathrm{O}_{3}$ showed no significant improvement upon further increase of reaction time. In other photodecomposition experiments, direct UV photolysis of $0.10 \mathrm{mM}$ TBBPA under alkaline conditions led to $90 \%$ decomposition after $240 \mathrm{~min}^{20)}$. In contrast, in the case of photocatalytic $\mathrm{TiO}_{2}$ oxidation, TBBPA (10 mg L ${ }^{-1}$; alkaline media; $100 \mathrm{~mL}$ ) was decomposed by approximately $50 \%$ after 60 min of UV irradiation ${ }^{21)}$. In the catalytic method, an aqueous solution of $0.072 \mathrm{mM}$ TBBPA was $100 \%$ decomposed within about $50 \mathrm{~min}$ in the presence of the $\mathrm{Pd}$ (1.9 wt. \% $) / \mathrm{TiO}_{2}$ solid catalyst at $\mathrm{pH} 10.5^{22)}$. The decomposition of TBBPA $\left(\mathrm{TOC}=400 \mathrm{mg} \mathrm{L}^{-1}\right)$ with $\mathrm{Fe} / \mathrm{Ni}$ bimetallic catalysis showed $\sim 98 \%$ mineralization after $96 \mathrm{hr}^{23)}$. For comparison, the rate of decomposition of tetrabromobisphenol-A in the presence of microorganisms increased
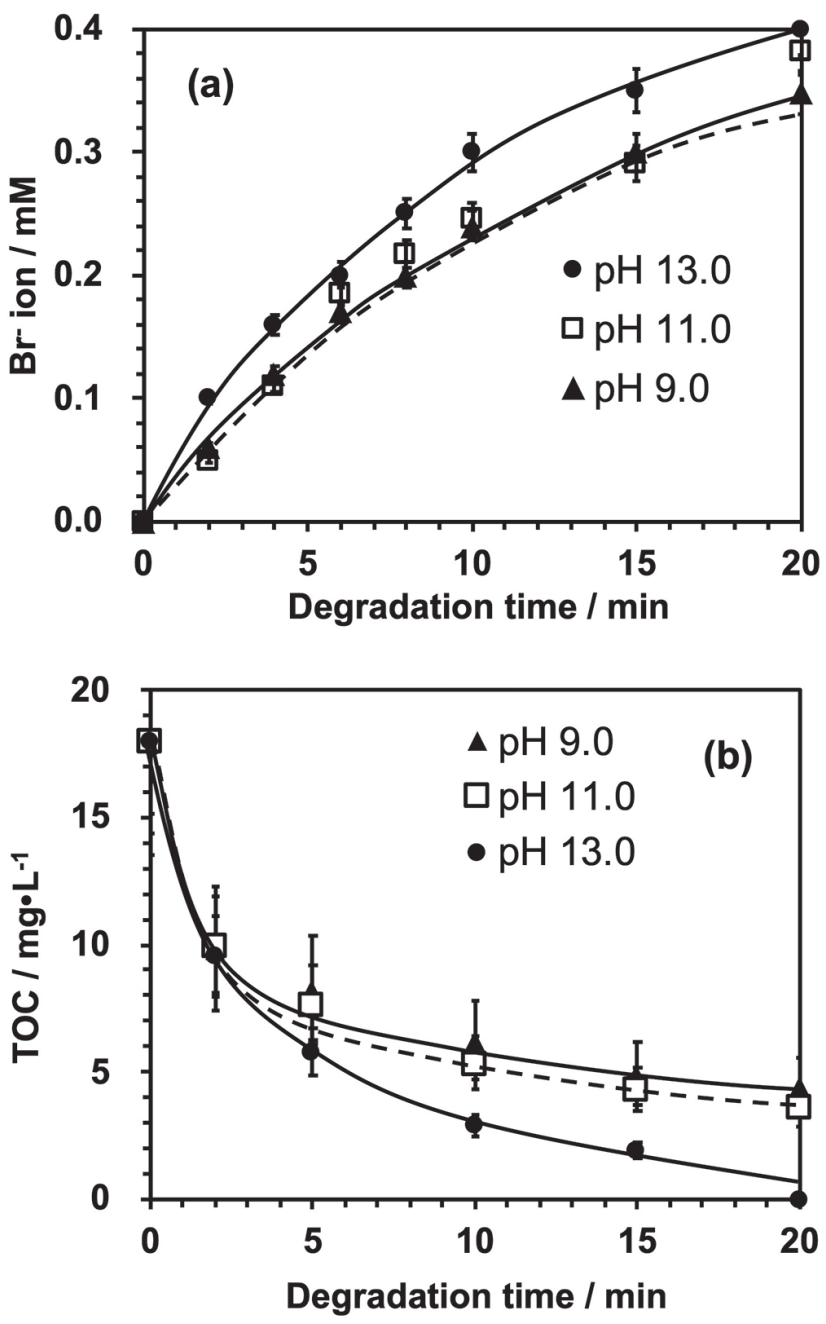

Fig. 2 (a) Dependence of the debromination of tetrabromobisphenol-A (TBBPA) at various alkaline $\mathrm{pHs}(\mathrm{pH}=9.0,11.0$ and 13.0) and on irradiation time; (b) plot showing the time course of the loss of total organic carbon (TOC) under plasma-inliquid irradiation at various pHs of 9.0, 11.0, and 13.0. Other conditions: volume of aqueous medium, $50 \mathrm{~mL}$; concentration of TBBPA, $0.10 \mathrm{mM}$ or ca. $54.4 \mathrm{mg} \mathrm{L}^{-1}$ (ppm); initial TOC concentration, 18.0 $\mathrm{mg} \mathrm{L}^{-1}$.

gradually with the biodegradation method; that is, the degradation experiment indicated that under non-domestic conditions, the degradation rate of $0.5 \mathrm{mg} \mathrm{L}^{-1} \mathrm{TBBPA}$ was $6.0 \%, 9.0 \%, 12.8 \%, 15.5 \%$, and $17.5 \%$ after $4,8,12,16$, and 20 days of treatment, respectively ${ }^{24)}$. Clearly, a comparison of the efficiencies of these earlier used methods in water treatment shows that the submerged MPL method presents considerable advantages. It is worth noting that microwave irradiation of the alkaline aqueous media increased the temperature from $25^{\circ} \mathrm{C}$ to $48^{\circ} \mathrm{C}$ after MPL had been generated in solution after $20 \mathrm{~min}$. Accordingly, we 
also investigated the thermal degradation of TBBPA at $48^{\circ} \mathrm{C}$, and confirmed that the flame retardant TBBPA underwent no thermally-induced degradation at this temperature.

\subsection{Intermediates formed in degradation of tetrabromo- bisphenol-A}

Within the present context, findings of the degradation of this flame retardant by various other techniques are also worth noting with regard to the intermediates and/or byproducts produced ${ }^{4-7)}$. In this regard, the photochemical transformation of aqueous TBBPA solution by UV irradiation at various $\mathrm{pH}$ values $\mathrm{pH}$ ranging from 5.5 to 10 , with process being most efficient at pH 9 and 10) was reported by Eriksson and co-workers ${ }^{4)}$, who identified some of the degradation products of TBBPA by GC-MS analysis. Three major degradation products were identified, namely 4-isopropyl-2,6-dibromophenol, 4-isopropylene-2,6-dibromophenol, and 4-(2-hydroxyisopropyl)-2,6-dibromophenol, together with minor amounts of 4-hydroxy-2,6-dibromophenol and 2,6-dibromophenol, and to a lesser extent 2(2,4-cyclopentadienyl)-2-(3,5-dibromo-4-hydroxyphenyl) propane.

Han and co-workers ${ }^{5)}$ examined the photosensitized oxidation of TBBPA involving singlet molecular oxygen $\left({ }^{1} \mathrm{O}_{2}\right)$ produced under visible light with Rose Bengal or methylene blue as the ${ }^{1} \mathrm{O}_{2}$ photosensitizers, where TBBPA was a ${ }^{1} \mathrm{O}_{2}$ quencher. The authors observed two major radicals being generated from the degradation of TBBPA upon reaction with ${ }^{1} \mathrm{O}_{2}$ in aqueous alkaline media $(\mathrm{pH}=10)$. One such species was the 2,6-dibromo-p-benzosemiquinone radical, while the second radical was likely 2,6-dibromo- $p$ benzosemiquinone containing an EPR-silent substituent at the 3-position ${ }^{5}$. Spin trapping with 5,5-dimethyl-1-pyrroline $N$-oxide (DPMO) showed that other minor radicals (either $\cdot \mathrm{OH}$ and/or carbon-centered) were also generated during the reaction of TBBPA with ${ }^{1} \mathrm{O}_{2}$.

By comparison, Qu et al. ${ }^{6}$ found that TBBPA at an initial concentration of $100 \mathrm{mg} \mathrm{L}^{-1}$ degraded after 6 min of ozonation at $\mathrm{pH}$ 8.0; however, the degradation was most efficient in aqueous alkaline media at $\mathrm{pH}$ values 9,10 , and 11 after about 6 min of ozonation. Seventeen reaction intermediates and by-products were identified using an electrospray time-of-flight mass spectrometer, with the first one observed being 2,4,6-tribromophenol. TOC reduction proceeded significantly slower, however, compared to the degradation of TBBPA. For instance, when TBBPA was completely decomposed within 6 min, only $9.8 \%$ of TOC was removed. At the end of a 120-min ozonation period, the extent of mineralization(i.e., loss of TOC) was a relatively low $22.0 \%$, which was attributed to the formation of recalcitrant by-products toward ozonation.

The generated MPL emitted white, yellow, and blue light (Fig. 1c). An analysis with a UV-visible spectrophotometer
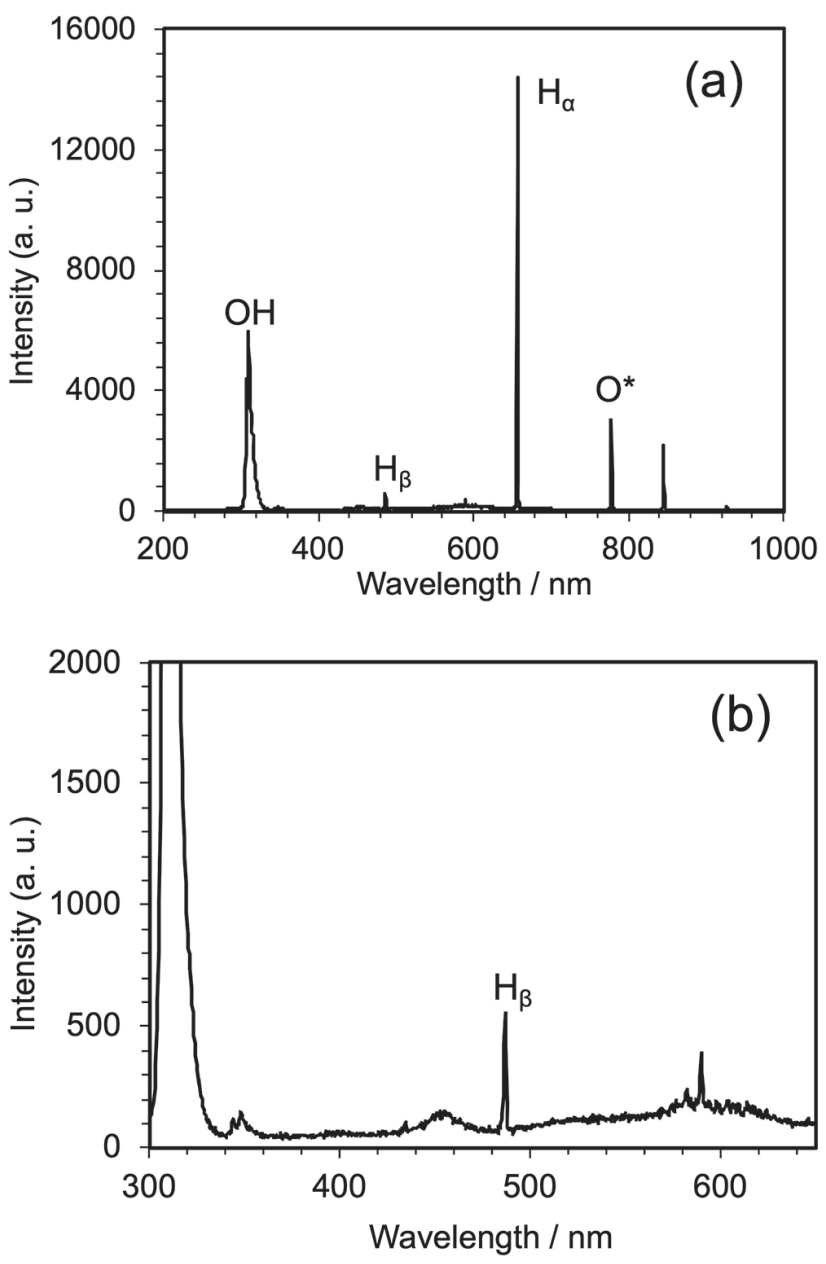

Fig. 3 (a) Spectrum of the light emitted from the plasmain-liquid generated in aqueous TBBPA solution, (b) expanded range from 300 to $700 \mathrm{~nm}$.

(Fig. 3) revealed relatively intense emission lines attributed to the formation of $\cdot \mathrm{OH}$ radicals $(316 \mathrm{~nm}), \mathrm{H}_{\alpha}$ hydrogen $(656 \mathrm{~nm})$, and $\mathrm{O}^{*}$ oxygen $(778 \mathrm{~nm})$. Peaks belonging to $\mathrm{H}_{\beta}$ hydrogen (Fig. 3b: $484 \mathrm{~nm}$ ) were likewise detected, albeit less intense. From the result of this spectrum, the MPL generated in the aqueous solution as the non-thermal plasma can generate $\cdot \mathrm{OH}\left(\mathrm{H}_{2} \mathrm{O} \rightarrow \mathrm{H} \cdot+\cdot \mathrm{OH}\right)$. Radical species are generated by water dissociation more effectively than by other conventional plasma technologies, because of the greater molecular density in liquid media than in gaseous media. Moreover, cavitation occurs when plasma is generated, as in ultrasonic methods. The reaction was likely initiated with the attack of reactive oxygen species (ROSs), such as $\cdot \mathrm{OH}$, produced from the generated MPL, although we cannot preclude the possible intervention of a photochemical route owing to the UV radiation produced by the plasma. Considering the former route, attack of the TBBPA substrate by $\cdot \mathrm{OH}$ likely occurs at atoms with the highest frontier electron densities, which were estimated using the MOPAC software system, and the results of 
Table 1 Calculations of frontier electron densities for tetrabromobisphenol-A: (a)chemical structure with atom number; (b) ranking of frontier electron densities.
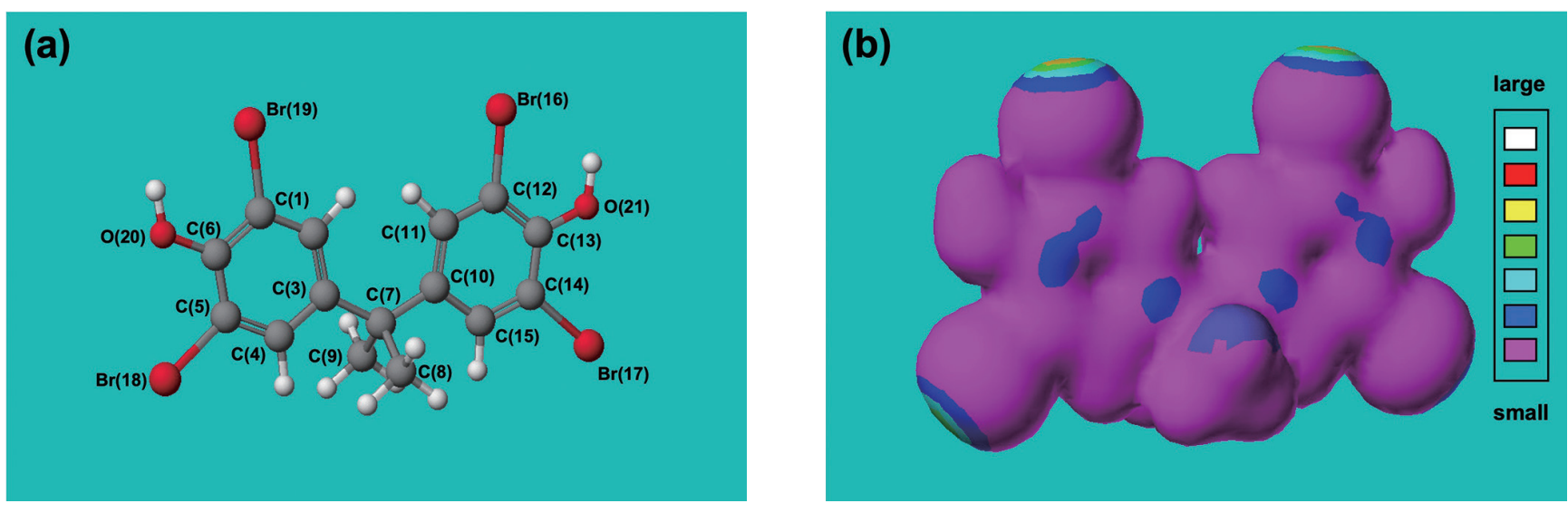

\begin{tabular}{|c|c|c|c|}
\hline Atoms & Frontier electron densities & Atoms & Frontier electron densities \\
\hline $\mathbf{C}(\mathbf{1})$ & $\mathbf{0 . 1 7 0}$ & $\mathrm{O}(20)$ & 0.057 \\
\hline $\mathrm{C}(2)$ & 0.060 & $\mathrm{O}(21)$ & 0.057 \\
\hline $\mathrm{C}(3)$ & 0.127 & $\mathrm{H}(22)$ & 0.001 \\
\hline $\mathrm{C}(4)$ & 0.063 & $\mathrm{H}(23)$ & $\sim 0$ \\
\hline $\mathbf{C}(\mathbf{5})$ & $\mathbf{0 . 1 3 7}$ & $\mathrm{H}(24)$ & 0.001 \\
\hline $\mathrm{C}(6)$ & 0.111 & $\mathrm{H}(26)$ & 0.001 \\
\hline $\mathrm{C}(7)$ & 0.009 & $\mathrm{H}(27)$ & $\sim 0$ \\
\hline $\mathrm{C}(8)$ & 0.012 & $\mathrm{H}(28)$ & 0.001 \\
\hline $\mathrm{C}(9)$ & 0.012 & $\mathrm{H}(29)$ & 0.001 \\
\hline $\mathrm{C}(10)$ & 0.127 & $\mathrm{H}(30)$ & 0.001 \\
\hline $\mathrm{C}(11)$ & 0.060 & $\mathrm{H}(31)$ & 0.001 \\
\hline $\mathbf{C}(\mathbf{1 2})$ & $\mathbf{0 . 1 7 0}$ & $\mathrm{H}(32)$ & 0.001 \\
\hline $\mathrm{C}(13)$ & 0.111 & $\mathrm{H}(34)$ & $\sim 0$ \\
\hline $\mathbf{C}(\mathbf{1 4})$ & $\mathbf{0 . 1 3 7}$ & & \\
\hline $\mathrm{C}(15)$ & 0.063 & & \\
\hline $\mathrm{Br}(16)$ & 0.143 & & \\
\hline $\mathrm{Br}(17)$ & 0.111 & & \\
\hline $\mathrm{Br}(18)$ & 0.111 & & \\
\hline $\mathrm{Br}(19)$ & 0.143 & & \\
\hline
\end{tabular}

which are reported in Table 1.

A perusal of Table 1 shows that along with the $\operatorname{Br}(16)$ and $\mathrm{Br}(19)$ atoms, the richest frontier electron densities in TBBPA were the $\mathrm{C}(1)$ and $\mathrm{C}(12)$ carbon atoms followed by the $\mathrm{C}(5)$ and $\mathrm{C}(14)$ carbon atoms, which were, therefore, most prone to attack by the $\cdot \mathrm{OH}$. In the present study, intermediates formed during the degradation of tetrabromophenol-A \{also known as 4,4' - (propane- 2,2-diyl)-bis (2,6-dibromophenol), (I); $m / z=544\}$ were identified by LC-MS spectra (see Fig. 4). Some of these intermediates (II) - (VIII) are illustrated in Fig. 5.

The three brominated intermediate products identified by LC-MS were 2,6-dibromo-4-[1-(3-bromo-4-hydroxyphenyl)-1- methylethyl]phenol(II), 1,1'-dihydroxy-2,2'-dibromobenzophenone (III), and 1,1'-dihydroxy-2-bromobenzophenone(IV). Subsequent to complete debromination, the intermediate 1,1'-dihydroxybenzophenone formed (V). Thereafter, opening of the benzene rings proceeded by further $\cdot \mathrm{OH}$ attack to yield the intermediates $(2 \mathrm{E}, 6 \mathrm{Z})$ -8-hydroxy-3-methyl-5-methylidene-4-oxoocta-2,6-dienal (VI), (3Z)-5-hydroxy-2-methylidenepent-3- enoic acid (VII), and 5-hydroxypentanoic acid (VIII), following which complete mineralization (that is, loss of TOC) ensued to yield $\mathrm{CO}_{2}$ gas and water. Thus, oxidative degradation took 

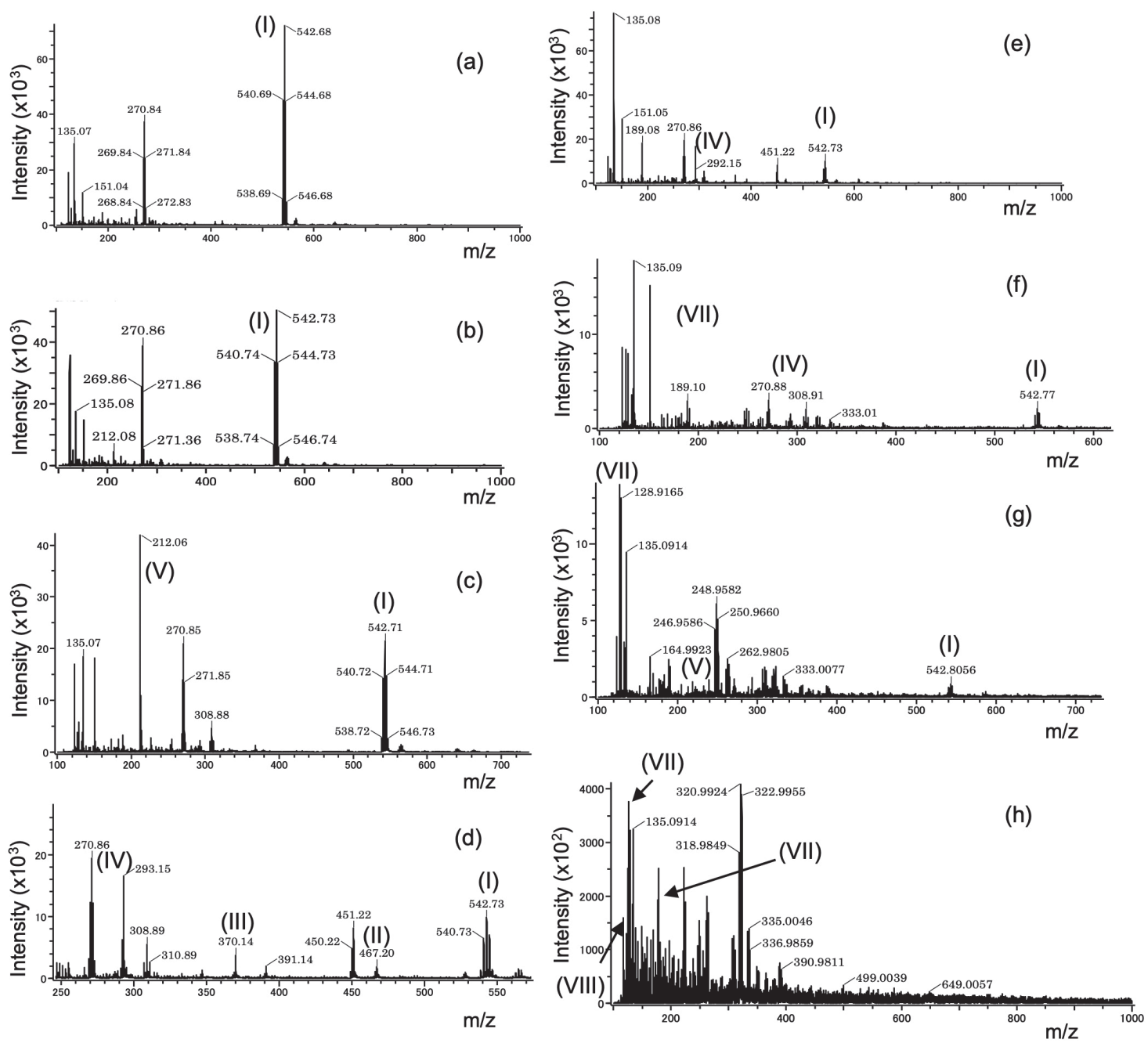

Fig. 4 Mass spectra recorded during the degradation of tetrabromobisphenol-A in pH 13.0 at different irradiation times:

(a) initial substrate (0 min), (b) 2 min, (c) 4 min, (d) 6 min, (e) 8 min, (f) $10 \mathrm{~min}$, (g) 16 min, and (h) $20 \mathrm{~min}$.<smiles>CC(C)(c1cc(Br)c(O)c(Br)c1)c1cc(Br)c(O)c(Br)c1</smiles>

(I)<smiles>CC(C)(c1ccc(O)c(Br)c1)c1cc(Br)c(O)c(Br)c1</smiles>

(II)<smiles>O=C(c1ccc(O)c(Br)c1)c1ccc(O)c(Br)c1</smiles>

(III)<smiles>O=C(c1ccc(O)cc1)c1ccc(O)c(Br)c1</smiles>

(IV)<smiles>O=C(c1ccc(O)cc1)c1ccc(O)cc1</smiles>

(V)<smiles>C=C(/C=C\CO)C(=O)C(C)=CC=O</smiles>

(VI)<smiles>C=C(/C=C\CO)C(=O)O</smiles>

(VII)<smiles>O=C(O)CCCCO</smiles>

(VIII)

Fig. 5 Proposed intermediates, (II)_(VIII), formed in the degradation of tetrabromobisphenol-A(I) occurring in aqueous media at $\mathrm{pH} 13.0$ by the microwave-induced plasma-in-liquid (MPL) method. 
place through debromination, hydroxylation, and cleavage of the aromatic rings to achieve TOC. Notably, debromination of TBBPA could also have occurred reductively by the plasma, followed by further decomposition by the $\cdot \mathrm{OH}^{12,14)}$ through opening of the benzene rings (Fig. 4), as also alluded to by other studies ${ }^{20,21,23)}$, albeit through different processes. Our LC-MS results likewise confirmed that there were no highly toxic by-products with Br substituents on polycyclic aromatic hydrocarbons (PAHs), in contrast to the report by Jin and co-workers ${ }^{7)}$.

\section{Conclusions}

We showed that the flame retardant TBBPA is decomposed and mineralized in a relatively short time under energy-saving conditions using the MPL method that we propose as another AOP process, adding to the family of AOPs in current use. The LC-MS results confirm that no contaminant intermediates, in which multiple Br substituents are bonded to the chemical structure, were generated by the MPL, in contrast to the cases using other AOP methods. More importantly, the MPL method does not use chemicals that might otherwise cause secondary contaminations associated with decomposition. Consequently, this plasma method may be considered a green environmental purification technique.

\section{Acknowledgment}

We are grateful to the Japan Society for the Promotion of Science(JSPS) for financial support to SH through a Grant-in-aid for Scientific Research (No. 19K22316). We would also like to thank to Prof. Sato at Saitama Institute of Technology for technical support. One of us (NS) is grateful to the staff of the PhotoGreen Laboratory at the University of Pavia, Italy, for their continued hospitality.

\section{References}

1) Glaze, W.-H.; Kang, J.-W.; Chaplin, D.H. The chemistry of water treatment processes involving ozone, hydrogen peroxide and ultraviolet radiation. Ozone Sci. Eng. 9, 335-352 (2008).

2) Braun, A.-M.; Maurette, M.-T.; Oliveros, E. Photochemical Technology. J. Wiley \& Sons. New York, NY, ISBN 0-471-92652-3(1991).

3) Deng, Y.; Zhao, R. Advanced oxidation processes (AOPs) in wastewater treatment. Current Pollution Rep. 1, 167-176 (2015).

4) Eriksson, J.; Rahm, S.; Green, N.; Bergman, A.; Jakobsson, E. Photochemical transformations of tetrabro-
mobisphenol-A and related phenols in water. Chemosphere 54, 117-126(2004).

5) Han, S.-K.; Bilski, P.; Karriker, B.; Sik, R.H.; Chignell, C.F. Oxidation of flame retardant tTetrabromobisphenol-A by singlet oxygen. Environ. Sci. Technol. 42, 166-172 (2008).

6) Qu, R.; Feng, M.; Wang, X.; Huang, Q.; Lu, J.; Wang, L.; Wang, Z. Rapid removal of tetrabromobisphenol A by ozonation in water: Oxidation products, reaction pathways and toxicity assessment. PLOS ONE 10, e0139580 (2015).

7) Jin, H.; Kong, D.; Ji, Y.; Lu, J.; Zhou, Q. Degradation of tetrabromobisphenol $\mathrm{A}$ in heat activated persulfate oxidation process. RSC Adv. 6, 29718-29726(2016).

8) Hakk, H. A survey of tetrabromobisphenol-A. Second International Workshop on Brominated Flame Retardants, BFR. Stockholm University, Sweden(2001). See http://chm.pops.int/Portals/0/docs/from_old_website/ documents/meetings/poprc/submissions/Comments_2006/Selected.brominated.flame. retardants. pdf\#search $=\% 27$ Hakk + H. \% 2C $+2001 .+$ A + survey + of + tetrabromobisphenolA. + Second + International

+ Workshop + on + Brominated + Flame + Retardants \%2C + BFR\%2C + Stockholm + University \% 2C + Sweden. \%27

9) Malkoske, T.; Tang, Y.; Xu, W.; Yu, S.; Wang, H. A review of the environmental distribution, fate, and control of tetrabromo-bisphenol-A released from sources. Sci. Total Environ. 569-570, 1608-1617(2016).

10) Sun, F.; Kolvenbach, B.A.; Nastold, P.; Jiang, B.; Ji, R.P.; Corvini, F.X. Degradation and metabolism of tetrabromobisphenol-A(TBBPA) in submerged soil and soil plant systems. Environ. Sci. Technol. 48, 1429114299 (2014).

11) Clements, J.S.; Sato, M.; Davis, R.H. Preliminary investigation of pre-breakdown phenomena and chemical reactions using a pulsed high-voltage discharge in water. IEEE Trans. Ind. Appl. IA-23, 224-235 (1987).

12) Horikoshi, S.; Serpone, N. In-liquid plasma: a novel tool in the fabrication of nanomaterials and in the treatment of wastewaters. $R S C A d v$. 7, 47196-47218 (2017).

13) Namihira, T.; Sakai, S.; Yamaguchi, T.; Yamamoto, K.; Yamada, C.; Kiyan, T.; Sakugawa, T.; Katsuki, S. Electron temperature and electron density of underwater pulsed discharge plasma produced by solid-state pulsed-power generator. IEEE Trans. Plasma Sci. 35, 614-618(2007).

14) Horikoshi, S.; Sawada, S.; Sato, S.; Serpone, N. Microwave-driven in-liquid plasma in chemical and environmental applications. III. Examination of optimum microwave pulse conditions for prolongation of electrode lifetime, and application to dye-contaminated wastewater. Plasma Chem. Plasma Process. 39, 51-62 
(2019)

15) Yonezawa, T.; Čempel, D.; Nguyen, M.T. Microwave-induced plasma-in-liquid process for nanoparticle production. Bull. Chem. Soc. Jpn. 91, 1781-1798(2018).

16) Rahim, I.; Nomura, S.; Mukasa, S.; Toyota, H.; Decomposition of methane hydrate for hydrogen production using microwave and radio frequency in-liquid plasma methods. Appl. Thermal Eng. 90, 120-126 (2015).

17) Sakugawa, T.; Aoki, N.; Akiyama, H.; Ishibashi, K.; Watanabe, M.; Kouda, A.; Suematsu, K. A method of cyanobacteria treatment using underwater pulsed streamer-like discharge. IEEE Trans. Plasma Sci. 42, 794-798(2014).

18) Horikoshi, S.; Sato, S.; Abe, M.; Serpone, N. A novel liquid plasma AOP device integrating microwaves and ultrasounds and its evaluation in defluorinating perfluorooctanoic acid in aqueous media. Ultrason. Sonochem. 18, 938-942 (2011).

19) Horikoshi, S.; Serpone, N.; Yoshizawa, S.; Knowland, J.; Hidaka, H. Photocatalyzed degradation of polymers in aqueous semiconductor suspensions.: IV Theoretical and experimental examination of the photooxidative mineralization of constituent bases in nucleic acids at
Titania/water interfaces. J. Photochem. Photobiol. A: Chem. 120, 63-74(1998).

20) Guo, Y.; Zhou, J.; Lou, X.; Liu, R.; Xiao, D.; Fang, C.; Wang, Z.; Liu, J. Enhanced degradation of Tetrabromobisphenol-A in water by a UV/base/persulfate system: Kinetics and intermediates. Chem. Eng. J. 254, 538$544(2014)$.

21) Guo, Y.; Lou, X.; Xiao, D.; Xu, L.; Wang, Z.; Liu, J. Sequential reduction-oxidation for photocatalytic degradation of tetrabromobisphenol-A: kinetics and intermediates. J. Hazard. Mater. 241-242, 301-306 (2012).

22) Wu, K.; Zheng, M.; Han, Y.; Xua, Z.; Zheng, S. Liquid phase catalytic hydrodebromination of tetrabromobisphenol-A on supported Pd catalysts. Appl. Surf. Sci. 376, 113-120 (2016).

23) Peng, X.; Wang, Z.; Huang, J.; Pittendrigh, B.-R.; Liu, S.; Jia, X. Wong, P.-K., Efficient degradation of tetrabromobisphenol A by synergistic integration of $\mathrm{Fe} / \mathrm{Ni}$ bimetallic catalysis and microbial acclimation. Water Res. 122, 471-480 (2017).

24) Peng, X.; Wang, Z.; Wei, D.; Huang, Q.; Jia, X. Biodegradation of tetrabromobisphenol-A in the sewage sludge process. J. Environ. Sci. 61, 39-48(2017). 